\title{
Novedades en el tratamiento del cáncer de próstata metastático hormono-independiente
}

\author{
Tan W.
}

Genitourinary Oncology, Hematology, Oncology Mayo Clinic. Jacksonville, Florida.

Actas Urol Esp. 2007;31(6):680-685

\section{RESUMEN \\ NOVEDADES EN EL TRATAMIENTO DEL CÁNCER DE PRÓSTATA METASTÁTICO HORMONO-INDEPENDIENTE}

Objetivo: revisar los últimos avances en el tratamiento del cáncer de próstata hormono-independiente (CPHI).

Metodologia: revisión de los últimos resúmenes de comunicaciones y presentaciones en congresos, y de la literatura científica utilizando las siguientes palabras clave para búsquedas en Medline/PubMed: androgen independent/hormone refractory prostate cancer, novel treatment options. Revisión de ensayos clínicos en fase II y fase III.

Conclusión: dos estudios de gran trascendencia, -el SWOG 9916 y el Taxotere 327- han probado que la supervivencia de los pacientes con CPHI puede mejorar tras la administración intravenosa de docetaxel cada 3 semanas. La combinación de docetaxel y prednisona proporciona supervivencias de 19 meses, mientras que la asociación de mitoxantrona y prednisona se sigue de supervivencias de 16 meses. A pesar de esa diferencia, todavia es necesario mejorar el beneficio en términos de supervivencia, dado que el período libre de recidiva entre los respondedores es frecuentemente breve (6 meses), y los pacientes a menudo mueren por progresión tumoral. El satraplatino (un nuevo análogo del platino) utilizado en segunda línea ha proporcionado un beneficio adicional de 1,5 semanas libres de progresión. No obstante, es necesario desarrollar drogas menos tóxicas para mejorar significativamente la supervivencia.

Palabras clave: Cáncer de próstata hormono-refractario. Cáncer de próstata hormono-independiente. Nuevas opciones de tratamiento.

\section{ABSTRACT \\ NOVEL PROMISING TREATMENT OPTIONS FOR METASTATIC ANDROGEN INDEPENDENT PROSTATE CANCER}

Objective: Review the recent advances in the treatment of androgen independent prostate cancer (AIPC).

Methods: Review recent abstracts and literature utilizing Medline/PubMed using key words: androgen independent/hormone refractory prostate cancer, novel treatment options, Phase II, III trials and meeting abstracts/presentations.

Conclusion: Two pivotal trials SWOG (Southwest Oncology Group) study

9916 and Taxotere 327 have shown that survival can be improved in this population by administration of chemotherapy with docetaxel every three weeks intravenously. An overall survival of 19 months could be achieved with docetaxel/prednisone compared to16 months with mitoxantrone/prednisone. Despite this, there is a need to improve on this survival benefit because the relapse free survival among responders is often short (6 months) and patients often would have progression of their cancer leading to death. Satraplatin, a novel platinum analogue had been found to provide an additional 1.5 week progression free survival benefit in this population in the second line setting. There is however, a need to develop less toxic drugs that would improve survival significantly.

Keywords: Androgen independent/hormone refractory prostate cancer. Novel treatment options. 
$\mathrm{E}^{1}$ cáncer de próstata se encuentra entre los tumores malignos más comunes en el varón. En los Estados Unidos se diagnostican cada año cerca de 218.890 nuevos casos; cada año, mueren 27.050 hombres por esta causa ${ }^{1}$. Con frecuencia, en el momento del diagnóstico la enfermedad se encuentra en fase curable. Sin embargo, una vez que la enfermedad recurre y se producen metástasis, la enfermedad es a menudo fatal.

\section{DOCETAXEL: BENEFICIO PARA LA SUPERVIVENCIA CUANDO SE UTILIZA COMO PRIMERA LÍNEA DE TRATAMIENTO}

Dos estudios de gran trascendencia condujeron a la aprobación de docetaxel como tratamiento del CPHI metastático: el estudio SWOG (Southwest Oncology Group) 9916 y el estudio TAX 327. El estudio SWOG-9916 es un ensayo clínico randomizado que comparó docetaxel/estramustina con mitoxantrona y prednisona en el tratamiento de 674 pacientes con CPHI metastático. La combinación que incluyó docetaxel resultó superior a la de mitoxantrona y prednisona, con una mediana de supervivencia de 17,5 meses y 15,6 meses $(\mathrm{p}=0,02)$, respectivamente, para cada una de las combinaciones. La mediana de tiempo a la progresión de los pacientes tratados con la combinación docetaxel/estramustina (6,3 meses) resultó significativamente superior $(\mathrm{p}<0,001)$ a la alcanzada entre los tratados con mitoxantrona y prednisona (3,2 meses). Finalmente, las cifras de PSA descendieron significativamente más $(p<0,001)$ entre los tratados con docetaxel/estramustina (descenso del 50\% frente a un descenso del 27\% entre los tratados con mitoxantrona y prednisona). Sin embargo, no se produjeron diferencias entre los dos brazos de tratamiento en lo referente a la respuesta tumoral objetiva ${ }^{2}$.

El estudio TAX 327 randomizó 1.006 pacientes a 3 regimenes terapéuticos diferentes: docetaxel/prednisona cada tres semanas (régimen 1), docetaxel/prednisona semanal (régimen 2), y mitoxantrona/prednisona cada tres semanas. Los pacientes incluidos en el régimen 1 experimentaron una mejoría de la supervivencia significativa comparada con el régimen 2 (18,9 meses frente a 16,5 meses, respectivamente, $\mathrm{p}<0,009$ ).
De modo similar, el control del dolor (45\% frente al $32 \%$, para los pacientes sometidos a los regímenes 1 y 2 , respectivamente, $\mathrm{p}<0,01)$ y la calidad de vida ( $22 \%$ frente al $13 \%$, para los pacientes sometidos a los regímenes 1 y 2 , respectivamente, $\mathrm{p}=0.009$ ) resultaron superiores entre los pacientes incluidos en el régimen 1 (docetaxel/ prednisona cada tres semanas) ${ }^{3}$.

Una reciente actualización del estudio TAX 327 confirmó el beneficio en términos de supervivencia del régimen de docetaxel cada tres semanas en comparación con mitoxantrona/prednisona (18,9 meses frente a 16,5 meses, respectivamente, razón de probabilidad 0,76 ), a la vez que descartó el beneficio del tratamiento con docetaxel semanal (18,9 meses para la combinación de docetaxel/prednisona cada tres semanas frente a 17,4 meses para docetaxel semanal, razón de probabilidad 0,091). A los tres años, la supervivencia fue del 17,2\% para los pacientes tratados con docetaxel/prednisona cada tres semanas, frente al 12,8\% alcanzado entre los tratados con mitoxantrona/prednisona $(\mathrm{p}=0,005)^{4}$.

Los dos estudios mencionados más arriba demostraron una reducción del $20-24 \%$ en la mortalidad de los pacientes con CPHI.

\section{COMBINACIONES DE QUIMIOTERAPIA}

Hasta la fecha se han ensayado varias combinaciones de quimioterapia buscando mejorar la respuesta de PSA (prostate specific antigen) y la eficacia, pero ninguna ha demostrado efecto alguno sobre la supervivencia. Entre otras, se han estudiado mediante ensayos clínicos en fase II las combinaciones de docetaxel/vinorelbina, docetaxel/capecitabina y docetaxel/talidomida ${ }^{5-7}$. La capecitabina ha sido utilizada en combinación con docetaxel dada su potencial sinergia. Vaishampayan presentó en 2007 los resultados de un ensayo fase II como tratamiento de primera línea en pacientes con CPHI. En un estudio sobre 30 pacientes, la combinación de docetaxel (36 mg $/ \mathrm{m}^{2}$ en los días 1,8 y 15) y capecitabina $\left(1.250 \mathrm{mg} / \mathrm{m}^{2}\right.$ desde el $5^{\circ}$ día hasta el $18^{\circ}$ día, cada 28 días) se siguió de un descenso de los niveles de PSA igual o superior al 50\% en el $71 \%$ de los pacientes (19/27), y una tasa de respuesta objetiva del $50 \%(5 / 10)$ en pacientes con enfermedad mesurable. El régimen resultó tolerable, 
con un caso de neutropenia grado $4 \mathrm{y}$ dos casos de toxicidad dermatológica (síndrome mano-pie) ${ }^{8}$.

\section{OPCIONES DE SEGUNDA LÍNEA}

Las opciones de tratamiento de segunda línea para el CPHI son limitadas. Hasta la fecha, se han utilizado para ese propósito tratamientos hormonales, quimioterapia y tratamientos radiofarmacológicos, pero únicamente el satraplatino ha demostrado tener algún efecto sobre la supervivencia $^{9,10}$. En un estudio retrospectivo sobre pacientes que recibieron al menos dos regímenes de quimioterapia por $\mathrm{CPHI}$, un grupo recibió en primer lugar docetaxel mientras que el otro fue sometido a tratamiento con mitoxantrona/prednisona en primer término. La tasa de respuesta (descenso de PSA igual o superior al 50\%) del tratamiento en primera línea fue del $68,2 \%$ para docetaxel, y del 12,1\% para la combinación mitoxantrona/prednisona. La tasa de respuesta del PSA tras tratamiento en segunda línea fue del $60,6 \%$ para docetaxel, y del $5,9 \%$ para la combinación mitoxantrona/prednisona. La mediana de tiempo a la progresión fue significativamente superior $(\mathrm{p}=0,01)$ entre los pacientes tratados con docetaxel (17 semanas) en comparación con los tratados con mitoxantrona (10 semanas). Sin embargo, no se detectaron diferencias en la supervivencia global con independencia de la secuencia del tratamiento ${ }^{11}$. Oh et al. publicaron los resultados de un estudio fase II utilizando docetaxel y carboplatino como segunda línea de tratamiento. La combinación de docetaxel (60 $\mathrm{mg} / \mathrm{m}^{2}$ ) y carboplatino (AUC 4) cada 3 semanas se asoció a un descenso del PSA del 50\% en 6 de 34 pacientes $(18 \%)$, y a una tasa de respuesta objetiva del $14 \%{ }^{12}$.

El satraplatino es una variante del platino para administración oral que se ha ensayado en el tratamiento del cáncer de próstata. Los estudios preliminares han demostrado que la combinación de satraplatino y prednisona en pacientes con CPHI vírgenes de tratamiento mejora la supervivencia libre de progresión que proporciona la prednisona en monoterapia (5,2 meses frente a 2,5 meses, para la combinación de satraplatino y prednisona, y para la prednisona en monoterapia, respectivamente, $\mathrm{p}=0,023$ ); adicionalmente, la combinación de satraplatino y predni- sona se asoció a un descenso del PSA superior al alcanzado tras tratamiento con prednisona en monoterapia ( $33 \%$ frente a $9 \%, \mathrm{p}=0,046)$.

El ensayo preveía incorporar a 380 pacientes, pero finalmente sólo se incorporaron 50 pacientes dado que el estudio fue clausurado prematuramente por sus promotores ${ }^{13}$. Un ensayo multinacional de satraplatino y prednisona frente a prednisona como tratamientos de segunda línea ha completado la fase de captación de pacientes. Novecientos cincuenta pacientes fueron randomizados. Los pacientes tratados con satraplatino experimentaron una reducción del $40 \%$ en el riesgo de progresión en comparación con el grupo tratado con prednisona. La supervivencia libre de progresión fue de 11 semanas para los tratados con satraplatino, y de 9,7 semanas para los tratados con prednisona. A los 12 meses, el 17\% de los pacientes tratados con satraplatino no habian sufrido progresión de la enfermedad, mientras que el $7 \%$ de los pacientes tratados con prednisona no habían progresado al final de ese período de tiempo ${ }^{14}$.

\section{NUEVOS AGENTES TERAPÉUTICOS}

Los nuevos agentes terapéuticos representan drogas que deben de ser consideradas dada su potencialidad para eliminar patrones de crecimiento tumoral únicos o múltiples, lo que podría conducir a una regulación a la baja de múltiples receptores y de otros factores importantes para la angiogénesis, la proliferación y la apoptosis (Tabla 1).

\section{Bevacizumab y sorafenib}

La angiogenesis constituye un paso importante hacia la progresión y el desarrollo de metástasis. El bevacizumab es un anticuerpo monoclonal del VEGF (vascular endothelial growth factor, factor de crecimiento endotelial vascular) que está

Tabla 1. Ensayos clínicos con nuevos agentes actualmente en curso

\begin{tabular}{ll}
\hline SWOG & docetaxel/imatinib vs docetaxel \\
EORTC & docetaxel/oblimersan vs docetaxel \\
CALGB & docetaxel bevacizumab vs doctaxel \\
SWOG & docetaxel/atrasentan vs docetaxel \\
Novacea & docetaxel/calcitriol vs docetaxel \\
Nexos & docetaxel/GVAX vs docetaxel
\end{tabular}


siendo objeto de ensayos clínicos en pacientes con CPHI). El Cancer Leukemia Group B (CALGB, Grupo B de Leucemia Cáncer) estudió el efecto del bevacizumab en un ensayo clínico en fase II. En combinación con docetaxel/estramustina, la administración de bevacizumab indujo una respuesta de PSA del 77\%; la mediana de supervivencia libre de progresión fue de 10 meses $^{15}$. Con base en este interesante hallazgo, y en poderosos datos preclínicos, el CALGB está llevando a cabo un ensayo clínico en fase III comparando docetaxel con la combinación de docetaxel y bevacizumab (Tabla 2).

Tabla II. Novedades terapéuticas, respuesta de PSA y mediana de supervivencia

\begin{tabular}{lcc}
\hline Agente & $\begin{array}{c}\text { Respuesta de } \\
\text { PSA }\end{array}$ & $\begin{array}{c}\text { Mediana de } \\
\text { supervivencia } \\
\text { (meses) }\end{array}$ \\
\hline Docetaxel & $45 \%$ & 19 \\
Bevacizumab/ & $77 \%$ & ND \\
docetaxel & $33 \%$ & 14 \\
Satraplatino & $81 \%$ & 23 \\
Docetaxel/calcitriol & NA & 25 \\
Sipuleucel & NA & 26 \\
GVAC & & \\
ND: no disponible & & \\
\hline
\end{tabular}

El sorafenib ha sido aprobado recientemente para el tratamiento del cáncer renal metastático al haber quedado demostrado un beneficio en términos de tiempo a la progresión (24 semanas para los tratados con sorafenib frente a 12 semanas para los tratados con placebo). El sorafenib es un inhibidor de kinasa múltiple que inhibe los factores B-RAF, VEGF, C-kit y PDGF (platelet derived growth factor, factor de crecimiento derivado de las plaquetas). Debido a su potencial efecto sobre la angiogénesis, sus efectos han sido ensayados en 22 pacientes con $\mathrm{CPHI}$ incluidos en un estudio en fase II; en ningún caso se produjo un descenso del PSA del 50\%; sin embargo, dos pacientes experimentaron una mejoría muy notoria de sus gammagrafias óseas ${ }^{16}$.

\section{Calcitriol/docetaxel}

Con frecuencia, las líneas celulares de cáncer de próstata contienen receptores de vitamina $\mathrm{D}$.
La exposición de líneas de cáncer de próstata a la vitamina $\mathrm{D}$ induce la diferenciación celular y disminuye la proliferación; por ese motivo, el calcitriol a alta concentración (DN-101) se ha utilizado para tratar el CPHI en ensayos clínicos.

Un ensayo en fase II de docetaxel y calcitriol en pacientes con $\mathrm{CPHI}$ proporcionó una respuesta de PSA del $81 \%$. La mediana de tiempo a la progresión fue de 11,4 meses. Otro estudio fase II que comparó el efecto de docetaxel/calcitriol con docetaxel como agente único no pudo detectar diferencia alguna en términos de respuesta de PSA (variable resultado principal); la dosis de docetaxel utilizada fue de $36 \mathrm{mg} / \mathrm{m}^{2}$ semanal (tres de cada cuatro semanas) en lugar de la dosis aprobada de docetaxel $\left(75 \mathrm{mg} / \mathrm{m}^{2}\right.$ cada 21 días). Sin embargo, la supervivencia global fue de 16 meses para los pacientes tratados con docetaxel/placebo, y de 23 meses para los tratados con la combinación ${ }^{17}$. Para confirmar los resultados preliminares, se está llevando a cabo un ensayo clínico randomizado de docetaxel/DN-101 y docetaxel en pacientes con CPHI (el estudio ASCENT-2).

\section{Imatinib y docetaxel}

El PDGF es importante en la patogénesis de las metástasis óseas, y se expresa en el $80 \%$ de los pacientes con CPHI. Los datos preclínicos han detectado la actividad de imatinib sobre líneas celulares de cáncer de próstata. En un ensayo en fase II de imatinib en pacientes con recidiva bioquímica tras tratamiento definitivo, se detectó un descenso del 50\% del nivel de PSA en un paciente de los 19 tratados $^{18}$. Un ensayo randomizado de docetaxel en fase III no detectó diferencias en eficacia cuando se comparó imatinib con la combinación de docetaxel e imatinib ${ }^{19}$.

\section{Ixabepilona}

La ixabepilona es una epotilona sintética con actividad contra los microtúbulos; hasta la fecha, ha demostrado ser muy efectiva en modelos tumorales animales resistentes a los taxanos. Un ensayo fase I de ixabepilona como agente único, y asociada a estramustima, proporciono una respuesta del PSA del 69\% para la combinación de drogas, y del $48 \%$ para el tratamiento como agente único ${ }^{20}$. Como tratamiento de primera línea la 
respuesta del PSA fue del 39\%; el mayor número de modificaciones de dosis por toxicidad se produjo por toxicidad neurológica ${ }^{21}$.

\section{Atrasentan}

El atrasentan es un nuevo inhibidor de la endotelina. La endotelina es un agente importante en la progresión y proliferación del cáncer de próstata. Varios estudios en fase II y III han sido incapaces de demostrar una mejoría significativa en el tiempo a la progresión; sin embargo, un metanálisis ha identificado una tendencia hacia la mejoría del tiempo hasta la progresión (estimada en el 19\%), a la vez que una mejoría de los marcadores de enfermedad ósea ${ }^{22,23}$. Nelson et al presentaron recientemente los datos de un estudio en fase III comparando atrasentan con placebo en pacientes con CPHI no metastático; no se detectaron diferencias en el tiempo a la progresión entre los dos agentes empleados (764 y 671 días para los pacientes tratados con atrasentan y placebo, respectivamente, razón de probabilidad 0,915, $\mathrm{p}=0,288$ ); de modo similar, la mediana de supervivencia fue de 1.477 días para los pacientes tratados con atrasentan, y de 1.403 días para los tratados con placebo (razón de probabilidad 0,909, $p=0,176$ ). Sin embargo, se produjo una disminución significativa de la fosfatasa alcalina ósea (-1.51 ui/l en los tratados con atresentan, y $+2.2 \mathrm{ui} / 1$ en el grupo tratado con placebo, $\mathrm{p}=0,001)$, a la vez que el tiempo de duplicación del PSA se alargó ( $\mathrm{p}=0,031)$. Un estudio fase III en curso (el SWOG S0421) compara el efecto de la combinación docetaxel/prednisona frente a docetacel/prednisona/atrasentan en pacientes con $\mathrm{CPHI}^{24}$.

\section{Oblimersan}

El BCL-2 es un regulador de la apoptosis. Los oligonucleótidos antisentido son importantes en la inhibición de la progresión tumoral. Un estudio randomizado multicéntrico de la EORTC (el EORTC 30021) ha comparado docetaxel como agente único frente a la combinación de docetaxel y oblimersan. Se produjo una respuesta objetiva en el 24\% (5/21) de los tratados con la combinación de drogas, y en el 19\% (5/27) de los tratados con docetaxel como agente único. La respuesta del PSA fue del 37\% en los pacientes tra- tados con la combinación, y del 46\% en los tratados con docetaxel como agente único. Los datos relacionados con el tiempo a la progresión y la supervivencia global no se encuentran disponibles debido al insuficiente seguimiento ${ }^{25}$.

\section{Vacunas}

En el cáncer el sistema inmune no funciona adecuadamente. En la actualidad, se encuentran en investigación vacunas que persiguen restaurar la función inmunitaria. Se han desarrollado varios tipos de vacunas dirigidas a diferentes proteinas presentes en el cáncer de próstata tales como el PSA, la fosfatasa ácida prostática (FAP) y los antígenos de membrana prostática.

El sipuleucel (Provenge, APC 8015) está constituido por células dendríticas autólogas que han sido sometidas a una proteína recombinante de la FAP y del factor estimulante de las colonias de macrófagos y granulocitos. Las células se extraen de la sangre de los pacientes mediante leucoforesis, y posteriormente son tratadas y reinfundidas. Un ensayo clínico en fase III utilizando un grupo control con placebo, no demostró una mejoría significativa en el tiempo a la progresión (variable resultado principal del estudio); a los 36 meses, por el contrario, se detectó una mediana de mejora de la supervivencia de 4,5 meses (25,9 frente a 21,4 meses) ${ }^{26}$. Un ensayo clínico está analizando el efecto de sipuleucel y placebo en pacientes asintomáticos con CPHI Gleason 7 o inferior. La droga está siendo evaluada por la FDA (Federal Drug Administration).

El GVAX es una vacuna fabricada a partir de células tumorales enteras de dos líneas celulares de cáncer de próstata (LNCaP y PC-3) genéticamente modificadas para producir GM-CSF; a nivel sistémico, las células inmunitarias activadas se focalizan sobre las células tumorales modificadas, y las destruyen.

Dos estudios fase II en pacientes con CPHI demostraron una tendencia hacia la mejoría de las gammagrafías óseas y de la mediana de tiempo a la progresión ${ }^{27}$.

Un análisis de las variables pronósticas en los ensayos fase II de la GVAX en pacientes con CPHI metastático, comparados según el nomograma de Halabi, demostraron un incremento de 7 meses de tiempo hasta la progresión $(26,2 \text { vs } 19)^{28}$. 
La combinación de docetaxel con los nuevos agentes terapéuticos persigue mejorar la supervivencia de los pacientes con CPHI. En el futuro resultaría ideal desarrollar drogas mínimamente tóxicas y dirigidas específicamente contra grupos de genes y proteínas de cada individuo con cáncer. Los estudios más recientes con vacunas y con las nuevas drogas pueden representar el primer escalón en ese proceso.

\section{REFERENCIAS}

1. Jemal A, Siegel R, Ward E et al. Cancer statistics 2007. CA Cancer J Clinic 2007 Jan feb 57;(1)43-66.

2. Petrylak DP, Tangen CM, Hussain MH, Lara PN, Jones JA, Taplin ME, et al. Docetaxel and estramustine comparedwith mitoxantrone and prednisone for advanced refractory prostate cancer . N Engl Med 2004;351(15):1513-1520.

3. Tannock IF, de Wit R, Berry WR, et al. Docetaxel plus prednisone or mitoxantrone plus prednisone for advanced prostate cancer. N Engl J Med 2004;351(15):1502-1512.

4. Berthold DR, Pond D, DeWit R et al. Docetaxel plus prednisone or mitoxantrone plus prednisone for advanced prostate cancer. Updated survival of Tax 327. Proc Ann Am Soc Clin Oncol (prostate) 2007; Abs 147.

5. Armstrong AJ, Carducci MA. New drugs in advanced prostate cancer . Curr Opin Urol 2006;16(3):138-145.

6. Berthold MR, Moore MJ. Novel targets in prostate cancer. Expert Opin Ther Targets 2006;10(5):777-780.

7. Tan WW. Novel agents and targets in managing patients with metastatic prostate cancer. Cancer Control 2006;13(3) 194-198.

8. Vaishampayan UN, Santucci R, Heilbrun L et al. Phase II trial of docetaxel + capecitabine combination in metastatic androgen independent prostate cancer. Proc Am Soc Clin Oncol (prostate) 2007 (abs 239).

9. Ross RW, Kantoff PW. Hormone-refractory prostate cancer: Choosing the appropriate treatment option. Oncology 2007;21(2):185-193.

10. Berthold DR, Moore MJ . Targeting metastatic Prostate cancer: The search for innovative systemic therapies. Oncology 2006;20(14): 1787-1792.

11. Oh WK, Manola J, Babcic V et al. Response to second-line chemotherapy in patients with hormone refractory prostate cancer receiving two sequences of mitoxatrone and taxanes. Urol 2006;67(6) 1235-1238.

12. Oh WK, Jacobus S Ross R et al. A phase II trial of docetaxel plus carboplatin in hormone refractory prostate cancer (HRPC) patients who have progressed after prior chemotherapy Proc Am Soc Clin Onc (prostate) 2007 (abstract 238)

13. Sternberg CN, Whelan P, Hetherington J, Genitourinary Tract Group of the EORTC. et al. Phase III trial of satraplatin, an oral platinum plus prednisone vs prednisone alone in patients with hormone refratory prostate cancer. Oncology 2005;68(1);2-5.

14. Petrylak DP, Sartor O, Witjes F et al. A phase III, randomized, double-blind trial of satraplatin and prednisone vs placebo prednisone for patients with hormone refractory prostae cancer. Proc Am Soc Clin Oncol (prostate) 2007 Abs 145

15. Picus J, Halabi S, Rini BI et al. The use of bevacizumab with docetaxel and estramustine in hormone refractory prostate cancer: initial results of CALGB 9006 Proc Am Soc Clin Oncol 2003:22:393 abs 1578 .
16. Dahut WL, Scripture CD, Posadas EM Et al. Bony metastatic disease responses to Sorafenib(Bay 43-9006) independent of PSA in patients with metastatic androgen independent prostate cancer J Clin Oncol 2006;24:3089-3094 Abs 4506.

17. Beer TM, Ryan CW, Venner PM et al. Double-blinded randomized study of high-dose calcitriol plus docetaxel compared with placebo plus docetaxel in androgen-indepedent prostate cancer: a report from the ASCENT Investigators. J Clin Oncol 2007;25(6)669-674.

18. Lin AM, Rini BI Weinberg V et al. A Phase II trial of imatinib mesylate in patients with biochemical relapse of prostate cancer after definitive local therapy.BJU Int 2006;98(4)763-769.

19. Matthew P, Thall PF, Johnson MM et al. Preliminary results of a randomized placebo-controlled double-blind trial of weekly docetaxel combined with imatinib in men with androgen indepedent prostate cancer and bone metastatses 2006. J Clin Oncol 24 (18s),232S abstract 4562.

20. Galsky MD, Small EJ, OH WK et al. Multi-institutional randomized phase II trial of the epothilone B analog ixabepilone (BMS 247550) with or without estramustine phosphate in patients with progressive castrate metastatic prostate Cancer. J Clin Oncol 2005;23:1439-1446.

21. Hussain M, Tangen CM, Lara PN Jr et al. Ixabepilone (epothilone B analogue BMS-247550) is active in chemotherapy naive patients with hormone-refractory Prostate cancer: a Southwest Oncology group trial SWOG 0111. J Clin 2005;23:8724-8729.

22. Carducci MA, Padley RJ, Breul J et al. Effect of endothelin-A receptor blockade with atrasentan on tumor progression in men with hormone refractory prostate cancer. A randomized phase II, placebo controlled trial J Clin Oncol 2005;25:679-689.

23. Vogelzang NJ, Nelson JB, Schulman CC et al. Met-analysis of endothelin-A receptor blockade with atrasentan on tumor progression in men with hormone refractory prostate cancer $\mathrm{J}$ Clin Oncol 2005;23(16s) 393s.

24. Nelson JB, Chin JL, Lane W et al. Results of a Phase III randomized controlled trial of the safety and efficacy of atrasentan in men with non-metastatic HRPC. Proc Ann Am Soc Clin Oncol (prostate) 2007

25. Sternberg CN, Dumez H, Van Poppel H et al. Multicenter randomized EORTC trial 30021 of docetaxel + Oblimersan with hormone refractory prostate cancer (HRPC). Proc Am Soc Clin Oncol (prostate) 2007, Abs 144.

26. Small EJ, Schellhammer PF, Higano CS et al. Placebo-controlled phase III trial of immunologic therapy with sipuleucel-T (APC 8015) in patients with metastatic, asymptomatic hormone refractory prostate cancer J Clin Oncol 2006; 24(19):3089-3094.

27. Simmons JW, Sacks N. Granulocyte-macrophage colony-stimulating factor-transduced allogeneic cancer cellular immunotherapy; The Gvax ${ }^{\circledR}$ vaccine for prostate cancer. Urol Oncol 2006;24:419-424.

28. Small EJ, Higano CS, Smith D et al. Analysis of prognostic variables in phase II trails of GVAX vaccine for prostate cancer in metastatic hormone refractory prostate cancer. Proc Am Soc Clin Oncol. 2006; Abs 254

Correspondencia autor: Dr. W. Tan

Genitourinary Oncology, Hematology/Oncology Mayo Clinic

4500 San Pablo RD Jacksonville, Florida 32224

Tel.: 9049536153

E.mail autor: tan.winston@mayo.edu

Información artículo: Original 